\title{
Acquired Drug Resistance During the Turnaround Time for Drug Susceptibility Testing Impacts Outcome of Tuberculosis
}

\section{Jiahui Zhu}

Changning Center for Disease Control and Prevention

\section{Lina Davies Forsman}

Karolinska University Hospital; Karolinska Institutet

\section{Ziwei Bao}

The Fifth People's Hospital of Suzhou

\section{Yan Xie}

Zigong Center for Disease Control and Prevention

\section{Zhu Ning}

Zigong Center for Disease Control and Prevention

\section{Xuliang Li}

Fudan University School of Public Health

\section{Sven Hoffner}

Karolinska Institutet: Department of Global Health

Yi Hu ( $\nabla$ yhu@fudan.edu.cn )

Fudan University School of Public Health

\section{Research Article}

Keywords: Acquired drug resistance, Phenotypic drug-susceptibility testing, Sputum culture conversion, Treatment failure

Posted Date: September 7th, 2021

DOl: https://doi.org/10.21203/rs.3.rs-850449/v1

License: (c) (i) This work is licensed under a Creative Commons Attribution 4.0 International License. Read Full License 


\section{Abstract}

\section{Background}

The impacts of acquired resistance to first-line drugs (FLDs) during turnaround time (TAT) for drug susceptibility testing (DST) are still unclear. Thus, we aimed to investigate the impacts of acquired resistance to FLDs during TAT for DST on tuberculosis (TB) standard treatment.

Method

We performed a prospective cohort study between 2013 and 2018 in China, including sputum culturepositive TB patients with a baseline DST result for a Mycobacterium tuberculosis (Mtb) isolate collected at TB diagnosis and a follow-up DST result for a Mtb isolate collected when baseline DST result became available. Mtb isolates with acquired drug resistance were identified by the comparison between baseline and follow-up DST. Treatment outcome were evaluated by sputum culture conversion and World Health Organization (WHO) treatment outcome definitions.

Results

In total, 65 patients with Mtb isolates with acquired resistance to any FLDs and 130 patients with consistent drug susceptibility profile were included in the analysis. In a Cox proportional hazard regression analysis, acquired pyrazinamide-resistance (aHR $0.54,95 \% \mathrm{Cl}: 0.36-0.81)$ and acquired isoniazid-resistance (aHR 0.50, 95\% Cl: 0.29-0.85) were associated with prolonged time to sputum culture conversion. Furthermore, independent risk factors of treatment failure included acquired INH-resistance (aOR 7.64, 95\%Cl: 2.39-16.08) and acquired PZA-resistance (aOR 5.71, 95\% Cl: 2.31-14.12).

Conclusion

The association between acquisition of drug resistance and treatment outcome highlights the importance of shortening the turnaround time of DST.

\section{Introduction}

Globally, an estimated 10.0 million (range, 8.9-11.0 million) people fell ill with tuberculosis (TB) in 2019(1). In the shadow of the current Covid-19 pandemic, where health services are disrupted and less patients screened and treated for $\mathrm{CB}_{\text {, }}$, the global number of TB deaths could increase by around $0.2-$ 0.4 million in 2020 alone(1).

Drug-resistant TB, especially multidrug-resistant or rifampicin-resistant TB (MDR/RR-TB) threatens global TB care and prevention and remains a major public health concern. Globally in 2019, 3.3\% of new TB cases and $17.7 \%$ of previously treated TB were MDR/RR-TB(1). For decades, diagnosis of drug-resistance has relied entirely on phenotypic drug susceptibility testing (DST), which routinely takes up to 6-8 weeks(2). During the two month-long turnaround time (TAT) for DST, drug susceptibility profiles of the 
Mycobacterium tuberculosis (Mtb) isolates might change due to suboptimal drug regimens, inadequate drug supply, low drug exposure or transmission of drug-resistant Mtb strains $(3,4)$. It's well known that acquired RIF-resistance leads to unfavorable outcome in TB patients, but little is known how the acquired resistance to isoniazid (INH), pyrazinamide (PZA) and ethambutol (EMB) impacts the treatment outcome $(5,6)$. Thus, we performed a prospective cohort study including three provinces in China to investigate the impacts of acquired resistance of the three first-line drugs (FLDs), INH, PZA and EMB, during the TAT for DST on TB treatment.

\section{Method}

Study population

We performed a prospective cohort study including three study provinces (Sichuan, Guizhou, and Zhejiang) in China. Midwestern Sichuan and its neighbor Guizhou to the southwest have two of the highest TB incidences in China, with 63 TB cases per 100,000 inhabitants and 122/100,000 respectively in 2018. The eastern province of Zhejiang has a lower TB incidence (33.52/100,000 in 2018). All the provinces have a similar three-tier health system consisting of village health stations, township, and county hospitals, as well as at least one TB-specialized health facilities in county level for TB diagnosis, treatment and case management. Due to limited local laboratory resources, bacterial cultures are sent to a prefectural TB reference laboratory for drug susceptibility testing (DST). All prefectural TB reference laboratories and hospital TB laboratories are quality assured in TB microbiological examinations at least twice yearly by the provincial TB reference laboratory. The study enrolled culture-confirmed pulmonary TB patients with a baseline DST result of a Mtb collected at TB diagnosis and a follow-up DST result of a Mtb isolate collected when baseline DST results were available in 2013-2018. All subjects were recruited in TB designated hospitals and received first-line anti-TB drugs standard treatment.

\section{Treatment and follow-up}

All TB patients received first-line drugs standard treatment after TB diagnosis that was made by smear microscopy, culture or WHO-recommended rapid diagnostic test. Standard first-line treatment for newly treated TB was 6 months of 2HRZE/4HR according to WHO recommendations(7). The former WHO recommendations of a routinely prolonged 8 months retreatment regimen was still in practice in China at the time of the study(8), including 2-month intensive phase, 1-month continuation period of INH, RIF, PZA and EMB, 5-month continuation period of INH, RIF and EMB. Hospitalization with directly observed therapy was recommended for the first two weeks of treatment. After discharge, drugs were supplied monthly basis and supervised by health care worker or family member. Follow-up clinical visits with collection of sputum samples were scheduled monthly during the 6-month or 8-month treatment duration.

Data collection

Information was collected regarding i) socio-demographic information: age, sex, place of residency; ii) clinical information: TB history (previously treated TB), disease severity on chest X-ray using the Timika 
score(9); iii) bacteriological diagnosis: sputum smear microscopy (positive or negative), sputum culture (positive or negative), drug susceptibility to the first-line drugs RIF, INH, PZA and EMB (susceptible or resistant). As the aim of the study was to study the impact of acquired drug resistance during TB treatment with first-line drugs, all cases of RIF-resistant Mtb was excluded from the analysis, since MDRTB treatment was provided.

Drug susceptibility testing

In the study, sputum samples were sent for Mtb culture using BACTEC MGIT ${ }^{\mathrm{TM}}$ (Becton, Dickinson and company, USA), according to the manufacture recommendations. Baseline sputum samples were sent for smear microscopy for TB diagnosis. At the same day, the duplicates of baseline sputum samples were sent to the regional TB reference labs for culture and phenotypic DST for four first-line drugs, INH, RIF, PZA and EMB (baseline DST). When baseline DST results became available, a sputum sample from each patient were collected for culture and follow-up DST to divide patients into groups: patients with acquired drug resistant Mtb isolates and patients with Mtb isolates with unchanged drug susceptibility profile. According to WHO recommendations(10), the critical concentrations of INH $0.1 \mathrm{mg} / \mathrm{L}, \mathrm{RIF} 1.0 \mathrm{mg} / \mathrm{L}, \mathrm{PZA}$ $100 \mathrm{mg} / \mathrm{L}$ and EMB $4 \mathrm{mg} / \mathrm{L}$ were used to classify the strains as susceptible or resistant. For quality assurance, the DST was repeated for $10 \%$ of the isolates by an external technician from Shanghai Municipal Center for Disease Control and Prevention who had passed the WHO's external quality control assurance with a consistency of over $95 \%$. No discrepancies were recorded.

\section{Definitions}

Disease severity of the patient's pulmonary infection was based on chest X-ray, summarized by the Timika score scale, where a score $>70$ is considered severe TB disease(9). New cases were defined as patients without history of prior TB treatment or who received less than one month of TB drugs. TAT for DST was defined as the duration from baseline sputum collection for culture up until phenotypic DST availability of baseline isolate. Acquired drug resistance was defined as new drug resistance (during the TAT for DST) in the isolate collected at the point of base-line DST completed, that was not present in the base-line isolate. For each patient with acquired drug resistant Mtb isolate, the time point of acquired drug resistance was the intermediate time point of TAT for DST. For patients with unchanged drug susceptibility profile, the starting time of observation was the intermediate time point of TAT for DST. Time to sputum culture conversion was defined as the duration from the intermediate time point of TAT for DST to the first time point when sputum culture negative for Mtb isolates, followed by three consecutive negative cultures. Successful treatment outcome was defined as "cure" or "treatment completed. Cure was defined as smear- or culture-negativity in the last month of treatment (and at least one previous occasion) and treatment completion if the patient had completed the 6- or 8-standard treatment, without fulfilling the aforementioned definitions of smear/culture-negativity(11). According to WHO recommendations (4th ed), if a patient whose sputum smear or culture was positive at 5 months or later during standard treatment, the treatment outcome was denoted as failure(11). 


\section{Statistical Analysis}

Data were collected using standardized forms, computerized and analyzed with R software (Version 1.2.1335). Log-rank test was used to evaluate the difference of the time to sputum culture conversion between patients with acquired drug resistance and those without. A Cox proportional hazard regression model was used to evaluate the difference of time from the intermediate TAT for DST to sputum culture conversion between patients with acquired drug-resistant Mtb isolates and patients with unchanged drug susceptibility profile by calculating hazard ratios (HRs) and $95 \%$ confidence intervals $(95 \% \mathrm{Cl})$. Logistic regression model was used to analyze the association between acquired drug resistance during TAT for DST and treatment failure. Age, sex and living areas were included as covariates in the adjusted model. Statistical tests were two-tailed with a significance level set at $p<0.05$.

\section{Results}

Characteristics of patients

A total of 1,479 patients were diagnosed with cultured-confirmed pulmonary TB patients who had Mtb isolates with baseline DST results during the study period in the study sites. Of these, 144 patients with MDR-TB were excluded, 42 patients lost to follow-up, and 1,094 patients were sputum culture negative when baseline DST results were available. A total of 199 patients had Mtb isolates with follow-up DST results. Comparing follow-up DST results with baseline DST results, 72 patients with Mtb isolates with acquired resistance to any of the four first-line drugs (RIF: 6, INH: 14, PZA: 34, EMB:3, INH and PZA combined: 13, EMB and PZA combined: 1, EMB and RIF combined:1) during TAT for DST. The seven cases of acquired RIF-resistant TB were excluded and the patients switched to MDR-TB treatment. Finally, 65 patients with Mtb isolates with acquired resistance to INH, PZA or EMB (INH: 14, PZA: 35, EMB:3, INH and PZA combined: 13, EMB and PZA combined: 1) and 127 patients with unchanged drug susceptibility profile during TAT (median 67.0 days, IQR 58.0-77.0 days) for DST, remained in analysis (Fig. 1). The median age was 46.0 (IQR 35.0-56.0) years, with a higher proportion of male patients $(67.6 \%)$ than females. The majority of patients $(72.4 \%)$ were newly treated cases and received 6-months standard treatment. Out of the 192 patients, $67.7 \%$ were susceptible to first-line drugs at TB diagnosis (Table 1 ). 
Table 1

Characteristics of patients

\begin{tabular}{|c|c|c|c|c|}
\hline \multirow[t]{3}{*}{ Characteristics } & \multirow{3}{*}{$\begin{array}{l}\text { Total } \\
\text { n (\%) }\end{array}$} & \multicolumn{2}{|c|}{ Drug-resistant profiles } & \multirow{3}{*}{$\begin{array}{l}P \\
\text { Value }\end{array}$} \\
\hline & & $\begin{array}{l}\text { Acquired drug- } \\
\text { resistance }\end{array}$ & Unchanged & \\
\hline & & n (\%) & n (\%) & \\
\hline Age/years (IQR) & $\begin{array}{l}46.0(35.0- \\
56.0)\end{array}$ & $46.0(37.0-56.0)$ & $\begin{array}{l}45.0(33.0- \\
56.0)\end{array}$ & $0.65^{\mathrm{a}}$ \\
\hline $\begin{array}{l}\text { TAT for DST/days } \\
\text { (IQR) }\end{array}$ & $\begin{array}{l}67.0(58.0- \\
77.0)\end{array}$ & $67.0(57.0-72.0)$ & $\begin{array}{l}68.0(59.0- \\
81.0)\end{array}$ & $0.20^{\mathrm{a}}$ \\
\hline \multicolumn{5}{|l|}{ Sex } \\
\hline Female & $62(32.3)$ & $18(29.0)$ & $44(33.8)$ & \\
\hline Male & $130(67.7)$ & $44(71.0)$ & $86(66.2)$ & 0.51 \\
\hline \multicolumn{5}{|l|}{ Place of residence } \\
\hline Zhejiang & $75(39.1)$ & $24(38.7)$ & $51(39.2)$ & \\
\hline Sichuan & $66(34.3)$ & $22(35.5)$ & $44(33.8)$ & \\
\hline Guizhou & $51(26.6)$ & $16(25.8)$ & $35(26.9)$ & 0.97 \\
\hline \multirow{2}{*}{\multicolumn{5}{|c|}{$\begin{array}{l}\text { Disease severity on } \\
\text { chest X-ray }\end{array}$}} \\
\hline & & & & \\
\hline Moderate & $156(81.3)$ & $49(79.0)$ & $107(82.3)$ & \\
\hline Severe & $36(18.8)$ & $13(21.0)$ & $23(17.7)$ & 0.59 \\
\hline \multicolumn{5}{|l|}{ TB history } \\
\hline Newly treated & 139 (72.4) & $47(75.8)$ & $92(70.8)$ & \\
\hline Previously treated & $53(27.6)$ & $15(24.2)$ & $38(29.2)$ & 0.47 \\
\hline \multicolumn{5}{|l|}{ Diabetes } \\
\hline No & $175(91.1)$ & 57 (91.9) & $118(90.8)$ & \\
\hline Yes & $17(8.9)$ & $5(8.1)$ & $12(9.2)$ & 0.79 \\
\hline \multicolumn{5}{|l|}{$\begin{array}{l}\text { Baseline drug- } \\
\text { resistance }\end{array}$} \\
\hline Susceptible & $130(67.7)$ & $26(41.9)$ & $39(30.0)$ & \\
\hline Resistant & $62(32.3)$ & $36(58.1)$ & $91(70.0)$ & 0.10 \\
\hline
\end{tabular}




\begin{tabular}{|c|c|c|c|c|}
\hline \multirow[t]{2}{*}{ Characteristics } & \multirow{2}{*}{$\begin{array}{l}\text { Total } \\
\text { n (\%) }\end{array}$} & \multicolumn{2}{|c|}{ Drug-resistant profiles } & \multirow{2}{*}{$\begin{array}{l}P \\
\text { Value }\end{array}$} \\
\hline & & $\begin{array}{l}\text { Acquired drug- } \\
\text { resistance } \\
\mathrm{n}(\%)\end{array}$ & $\begin{array}{l}\text { Unchanged } \\
\text { n (\%) }\end{array}$ & \\
\hline \multicolumn{5}{|c|}{$\begin{array}{l}\text { Data were presented by numbers (\%), unless otherwise indicated. IQR, Inter-quartile range; TB, } \\
\text { Tuberculosis; TAT for DST, turnaround time for drug-susceptibility testing; Diseases severity on chest } \\
\text { X-ray was defined by Timika score; Severe disease, Timika score }>71 ; P^{a} \text { were calculated by Mann- } \\
\text { Whitney test. }\end{array}$} \\
\hline
\end{tabular}

Impact of acquired drug resistance on time to sputum culture conversion

Acquired drug resistance during TAT for DST was associated with a significantly lower chance of sputum culture conversion, compared to patients with unchanged drug-susceptibility profile $(78.5 \%$ vs $94.5 \%, p<$ 0.001). Moreover, the Kaplan-Meier analysis revealed the median time to sputum culture conversion in patients whom Mtb isolates with acquired PZA-resistance was 15 days, which was close to that of patients whom Mtb isolates with unchanged susceptibility to PZA (Fig. 2). After adjustment for age, sex, TB history, disease severity on chest $x$-ray and baseline drug susceptibility profile; acquired resistance to PZA (aHR 0.54, 95\% Cl: $0.36-0.81, p=0.01$ ) and acquired resistance to INH (aHR $0.50,95 \% \mathrm{Cl} 0.29-0.85$, $p=0.01$ ) were still significantly associated with prolonged time to sputum culture conversion (Table 2 ). 
Table 2

Cox proportional hazard regression analysis for sputum culture conversion

\begin{tabular}{|c|c|c|c|}
\hline Variables & $\begin{array}{l}\text { Sputum conversion } \\
\mathrm{n}(\%)\end{array}$ & $\mathrm{HR}(95 \% \mathrm{Cl})$ & Adjusted HR (95\%Cl) \\
\hline Age/years (IQR) & $45.0(34.0-56.0)$ & $1.00(0.99-1.01)$ & $1.00(0.99-1.01)$ \\
\hline \multicolumn{4}{|l|}{ Sex } \\
\hline Female & $56(90.3)$ & 1.00 & 1.00 \\
\hline Male & $115(88.5)$ & $0.98(0.71-1.35)$ & $1.01(0.73-1.40)$ \\
\hline \multicolumn{4}{|l|}{ Place of residence } \\
\hline Zhejiang & $61(35.7)$ & 1.00 & 1.00 \\
\hline Sichuan & $59(34.5)$ & $1.11(0.78-1.59)$ & $1.11(0.77-1.59)$ \\
\hline Guizhou & $51(29.8)$ & $1.72(0.81-1.70)$ & $1.15(0.79-1.67)$ \\
\hline \multicolumn{4}{|l|}{$\begin{array}{l}\text { Disease severity on } \\
\text { chest X-ray }\end{array}$} \\
\hline Moderate & $137(87.8)$ & 1.00 & - \\
\hline Severe & $34(94.4)$ & $1.23(0.84-1.80)$ & - \\
\hline \multicolumn{4}{|l|}{ TB history } \\
\hline Previously treated & $48(90.6)$ & 1.00 & - \\
\hline New cases & $123(88.5)$ & $1.10(0.77-1.54)$ & - \\
\hline \multicolumn{4}{|l|}{ Diabetes } \\
\hline No & $155(88.6)$ & 1.00 & - \\
\hline Yes & $16(94.1)$ & $1.12(0.67-1.87)$ & - \\
\hline \multicolumn{4}{|l|}{ Baseline drug-susceptibility } \\
\hline Susceptible & $119(91.5)$ & 1.00 & - \\
\hline Resistant & $52(83.9)$ & $0.96(0.70-1.32)$ & - \\
\hline \multicolumn{4}{|l|}{ Drug susceptibility profile } \\
\hline Unchanged & $120(94.5)$ & 1.00 & 1.00 \\
\hline Acquired drug resistance & $51(78.5)$ & $0.69(0.49-0.96)$ & $0.68(0.49-0.96)$ \\
\hline \multicolumn{4}{|l|}{$\mathrm{INH}$} \\
\hline Unchanged & $152(92.1)$ & 1.00 & 1.00 \\
\hline
\end{tabular}




\begin{tabular}{|c|c|c|c|}
\hline Variables & $\begin{array}{l}\text { Sputum conversion } \\
\text { n (\%) }\end{array}$ & $\mathrm{HR}(95 \% \mathrm{Cl})$ & Adjusted HR (95\%Cl) \\
\hline Acquired resistance & $19(70.4)$ & $0.56(0.34-0.93)$ & $0.50(0.29-0.85)$ \\
\hline \multicolumn{4}{|l|}{ PZA } \\
\hline Unchanged & $137(95.1)$ & 1.00 & 1.00 \\
\hline Acquired resistance & $34(70.8)$ & $0.56(0.38-0.83)$ & $0.54(0.36-0.81)$ \\
\hline \multicolumn{4}{|l|}{ EMB } \\
\hline Unchanged & 167 (88.8) & 1.00 & 1.00 \\
\hline Acquired resistance & $4(100.0)$ & $1.15(0.43-3.11)$ & $1.09(0.40-3.00)$ \\
\hline \multicolumn{4}{|c|}{$\begin{array}{l}\text { Data were presented by numbers (\%), unless otherwise indicated. IQR, Inter-quartile range; TB, } \\
\text { Tuberculosis; Diseases severity on chest X-ray was defined by Timika score; Severe disease, Timika } \\
\text { score > 71; INH, isoniazid; PZA, pyrazinamide; EMB, ethambutol; HR and aHR were calculated by coX } \\
\text { proportional hazard regression model; Age, sex and place of residence were included as covariates in } \\
\text { the adjusted model. }\end{array}$} \\
\hline
\end{tabular}

Impact of acquired drug resistance during TAT for DST on treatment failure

A total of $17.7 \%$ (34/192) patients failed treatment. A significantly higher rate of treatment failure was seen among patients whom $\mathrm{Mtb}$ isolates with acquired drug resistance compared to patients who had isolates with unchanged drug susceptibility profile $(32.3 \%$ vs $10.2 \%, p<0.05)$. The multivariate logistic regression analysis revealed that acquired drug resistance was significantly associated with treatment failure (aOR 4.45, 95\% Cl 1.99-9.98, $p<0.05$ ). Acquired INH-resistance (aOR 7.64, 95\% Cl 2.39-16.08, $p<$ $0.05)$ and acquired PZA-resistance (aOR $5.71,95 \% \mathrm{Cl} 2.31-14.12, p<0.001)$ were significantly associated with treatment failure (Table 3). 
Table 3

Logistic regression analysis for treatment failure in TB patients

\begin{tabular}{|c|c|c|c|}
\hline Variables & $\begin{array}{l}\text { Treatment failure } \\
\mathrm{n}(\%)\end{array}$ & OR $(95 \% \mathrm{Cl})$ & Adjusted OR (95\% Cl) \\
\hline Age/years (IQR) & $48.0(38.0-57.0)$ & $1.02(0.99-1.04)$ & $1.02(0.99-1.05)$ \\
\hline \multicolumn{4}{|l|}{ Sex } \\
\hline Female & $11(17.7)$ & 1.00 & 1.00 \\
\hline Male & $23(17.7)$ & $0.99(0.45-2.20)$ & $0.96(0.40-2.30)$ \\
\hline \multicolumn{4}{|l|}{ place of residence } \\
\hline Zhejiang & $14(41.2)$ & 1.00 & 1.00 \\
\hline Sichuan & $9(26.5)$ & $0.87(0.37-2.08)$ & $0.83(0.33-2.08)$ \\
\hline Guizhou & $11(32.4)$ & $0.93(0.37-2.36)$ & $0.92(0.35-2.40)$ \\
\hline \multicolumn{4}{|l|}{$\begin{array}{l}\text { Disease severity on } \\
\text { chest x-ray }\end{array}$} \\
\hline Moderate & $32(20.5)$ & 1.00 & - \\
\hline Severe & $2(5.6)$ & $0.23(0.05-1.00)$ & - \\
\hline \multicolumn{4}{|l|}{ TB history } \\
\hline Previously treated & $9(17.0)$ & 1.00 & - \\
\hline New cases & $25(18.0)$ & $1.07(0.46-2.48)$ & - \\
\hline \multicolumn{4}{|l|}{ Baseline drug susceptibility } \\
\hline Susceptible & $9(13.4)$ & 1.00 & - \\
\hline Resistant & $25(20.0)$ & $1.27(1.02-1.59)$ & - \\
\hline \multicolumn{4}{|l|}{ Drug susceptibility profile } \\
\hline Unchanged & $13(10.2)$ & 1.00 & 1.00 \\
\hline Acquired drug resistance & $21(32.3)$ & $4.19(1.93-9.08)$ & $4.45(1.99-9.98)$ \\
\hline \multicolumn{4}{|l|}{$\mathrm{INH}$} \\
\hline Unchanged & $21(12.7)$ & 1.00 & 1.00 \\
\hline Acquired resistance & $13(48.1)$ & $6.37(2.63-15.40)$ & $7.64(2.39-16.08)$ \\
\hline \multicolumn{4}{|l|}{ PZA } \\
\hline Unchanged & $14(9.7)$ & 1.00 & 1.00 \\
\hline
\end{tabular}




\begin{tabular}{|c|c|c|c|}
\hline Variables & $\begin{array}{l}\text { Treatment failure } \\
\mathrm{n}(\%)\end{array}$ & OR $(95 \% \mathrm{Cl})$ & Adjusted OR (95\% Cl) \\
\hline Acquired resistance & $20(41.7)$ & $6.63(2.99-14.70)$ & $5.71(2.31-14.12)$ \\
\hline \multicolumn{4}{|l|}{ EMB } \\
\hline Unchanged & 33 (17.6) & 1.00 & 1.00 \\
\hline Acquired resistance & $1(25.0)$ & $1.57(0.16-15.53)$ & $2.14(0.19-23.92)$ \\
\hline \multicolumn{4}{|c|}{$\begin{array}{l}\text { Data were presented by number (\%), unless otherwise indicated. IQR, Inter-quartile range; TB, } \\
\text { tuberculosis; Diseases severity on chest X-ray was defined by Timika score; Severe disease, Timika } \\
\text { score > 71; INH, isoniazid; PZA, pyrazinamide; EMB, ethambutol; OR and aOR were calculated by } \\
\text { logistic regression model; Age, sex and place of residence were included as covariates in the adjusted } \\
\text { model. }\end{array}$} \\
\hline
\end{tabular}

\section{Discussion}

In this study, we found an alarmingly high rate $(33.9 \%, 65 / 192)$ of acquired drug resistance to three firstline drugs during TAT for DST. Acquired PZA-resistance and acquired INH-resistance during TAT for DST were associated with longer time to sputum culture conversion. Furthermore, acquired INH-resistance as well as acquired PZA-resistance during TAT for DST were significantly associated with increased risk of treatment failure.

In this study, 33.9\% (65/192) developed acquired resistance to the three first-line drugs, INH, PZA and EMB, during TAT for DST. Compared to our study with $33.9 \%$ acquired drug resistance, another crosssectional survey from a high TB burden area found that acquired drug resistance developed in $31.0 \%$ (19/62) Mtb isolates during first 2 months of treatment(12). A possible explanation for the acquired drug resistance is variable and sub-therapeutic drug exposure, which is commonly seen as highlighted by a previous study. Therapeutic drug monitoring is thus recommended in patients with risk factors for altered drug exposure, for instance malabsorption or diabetes(13). Our findings suggest that a large proportion of TB patients received inadequate treatment in China, which might hamper TB control. Optimized therapy based on timely and accurate DST results, especially in high drug-resistant TB burden areas, would be beneficial for TB treatment.

We found that acquired PZA-resistance and acquired INH-resistance during TAT for DST were independent predictors of longer time for sputum culture conversion. In a previous study, PZA-resistance as well as previously treated TB was linked with prolonged time to sputum culture conversion(14-16). Without the sterilizing activity of PZA, there is a risk of continued TB transmission and increased risk of relapse(17). Given the importance of early sputum culture conversion, individual treatment regimens coupled with interventions, such as regular surveillance to drug susceptibility profile of Mtb isolates, are necessary. 
Furthermore, acquired INH-resistance and acquired PZA-resistance were observed to be independently associated with treatment failure. The finding was in line with previous meta-analysis where treatment failure was significantly more common in patients with INH-resistant strains than in those infected with drug susceptible strain, when treated with first-line tuberculosis drugs(18). Therefore, the WHO treatment recommendation for INH resistant TB from 2017 should be applied (6 months RIF, EMB, PZA and a fluoroquinolone)(10). A cohort study also found that undiagnosed PZA-resistance might compromise treatment outcome of PZA containing drug regimens(19). Accordingly, if drug susceptibility profile of Mtb isolate changes during TAT for DST, physicians should promptly adjust and individualize treatment. Additionally, the higher treatment failure rate among patients with acquired INH- and PZA-resistance indicate the need for more vigilant clinical drug-resistance monitoring and timely repeat DST results during anti-TB treatment.

Relying on the systematic clinical investigation and microbiological testing, the present study made it possible to observe how acquired drug resistance during TAT for DST impact time to sputum culture conversion and treatment outcome. The fact that DST was performed in reference TB laboratories, including repeat DST, provides quality assurance. Meanwhile, the relatively large sample size made it possible to consider covariates and confounders in the multivariate analysis. The present study also have some limitations. Firstly, phenotypic DST for PZA using BACTEC MGIT ${ }^{\text {TM }}$ might be affected by some factors, such as bacterial activity and load, but WGS for genes associated with PZA-resistance was performed for strict quality control and the consistency rate reached more than $90 \%$. Secondly, the timing of acquired drug resistance were difficult to determine and thus we used the middle points of TAT for DST as an approximation. Moreover, the studied subjects received standardized regimens and some finding might be not generalizable to a population with high proportion of individualized treatment. However, our results support the use of an individualized treatment with follow-up DST in case of continued culture positivity, as the proportion of acquired drug resistance was significant. Furthermore, the routine use of a prolonged 8 month treatment regimen with first-line drugs without a renewed DST should be abolished, considering the risk of causing more drug resistance.

\section{Conclusions}

In conclusion, we found that acquired PZA-resistance during TAT for DST might prolong the time to sputum culture conversion as well as increase the treatment failure rate and acquired INH-resistance was also an independent risk factor for treatment failure. Our observation highlights the need for regular drugresistance monitoring and adjustment of treatment regimens based the changed drug susceptibility profile during TB treatment.

\section{Abbreviations}

FLDs: First-line drugs

TAT: Turnaround time 
DST: Drug-susceptibility testing

TB: Tuberculosis

Mtb: Mycobacterium tuberculosis

WHO: World health organization

HR: Hazard ratio

Cl: Confidence interval

OR: Odds ratio

MDR/RR-TB: Multidrug-resistant/Rifampicin-resistant tuberculosis

RIF: Rifampicin

INH: Isoniazid

PZA: Pyrazinamide

EMB: Ethambutol

\section{Declarations}

\section{Ethical approval and consent to participate}

This study was approved by the Public Health School of Fudan university (IRB\#2012-11-0245). All participants consented to participate and provided written informed consent.

\section{Consent for publication}

Not applicable.

\section{Availability of data and materials}

All data generated or analysed during this study are included in this published article.

\section{Competing interests}

The authors declare that they have no competing interests.

\section{Funding}

This work was supported by grants from Shanghai New Three-year Action Plan for Public Health (Grant No. GWV-10.1-XK16), the Science and technology project of Suzhou City Health Bureau (No. 
LCZX201918), Special project of diagnosis and treatment technology for clinical key diseases in Suzhou (LCZX201819), as well as Stockholm County Council (Davies Forsman dnr LS 2018-1256). The funders had no role in study design, data collection and analysis, decision to publish, or preparation of the manuscript.

\section{Authors' contributions}

Prof. Yi Hu conceptualised and designed the study. Yan Xie, Zhu Ning and Xuliang Li collected data and were involved in patient management. Jiahui Zhu performed data analysis and wrote the initial draft of the manuscript. Lina Davies Forsman, Ziwei Bao and Yi Hu reviewed the manuscript for important intellectual content. Sven Hoffner made some grammatical revisions. All authors read and approved the final manuscript.

\section{Conflict of Interest}

None to declare.

\section{Acknowledgments}

None to declare.

\section{Authors' information}

${ }^{1}$ Department of Public Health Monitoring and Management, Changning Center for Disease Control and Prevention, Shanghai, China. ${ }^{2}$ Department of Infectious Disease, Karolinska University Hospital, Stockholm, Sweden. ${ }^{3}$ Department of Medicine, Division of Infectious Diseases, Karolinska Institutet Solna, Sweden. ${ }^{4}$ The Fifth People's Hospital of Suzhou, Infectious Disease Hospital Affiliated to Soochow University, Suzhou, China. ${ }^{5}$ Department Tuberculosis Control, Zigong Center for Disease Control and Prevention, Zigong, China. ${ }^{6}$ School of Public Health and Key Laboratory of Public Health Safety, Fudan University, Shanghai, China. ${ }^{7}$ Department of Global Public Health, Karolinska Institutet, Stockholm, Sweden.

\section{References}

1. Global tuberculosis report 2020. Geneva: World Health Organization. 2020.

2. Van Cutsem G, Isaakidis P, Farley J, Nardell E, Volchenkov G, Cox H. Infection Control for DrugResistant Tuberculosis: Early Diagnosis and Treatment Is the Key. Clin Infect Dis. 2016;62 Suppl 3:S23843.

3. Chen Y, Yuan Z, Shen X, Wu J, Wu Z, Xu B. Resistance to Second-Line Antituberculosis Drugs and Delay in Drug Susceptibility Testing among Multidrug-Resistant Tuberculosis Patients in Shanghai. Biomed Res Int. 2016;2016:2628913. 
4. Nguyen VA, Nguyen HQ, Vu TT, Nguyen NA, Duong CM, Tran TH, et al. Reduced turn-around time for Mycobacterium tuberculosis drug susceptibility testing with a proportional agar microplate assay. Clin Microbiol Infect. 2015;21(12):1084-92.

5. Kuaban C, R Bercion, G Jifon, $P$ Cunin, KN BlackettBlackett. Acquired anti-tuberculosis drug resistance in Yaounde, Cameroon. Int J Tuberc Lung Dis. 2000;5(4):427-32.

6. Loutet MG, Davidson JA, Brown T, Dedicoat M, Thomas HL, Lalor MK. Acquired Resistance to Antituberculosis Drugs in England, Wales, and Northern Ireland, 2000-2015. Emerging infectious diseases. 2018;24(3):524-33.

7. Falzon D, Jaramillo E, Schünemann $\mathrm{H}$. The 2011 update of the World Health Organization guidelines for the programmatic management of drug-resistant tuberculosis. European Respiratory Journal. 2011;38(Suppl 55):393.

8. Association CM. Guidelines for primary diagnosis and treatment of tuberculosis (2018). Chinese Journal of General Practitioners. 2019;18(8):709-17.

9. Wejse C, Gustafson P, Nielsen J, Gomes VF, Aaby P, Andersen PL, et al. TB score: Signs and symptoms from tuberculosis patients in a low-resource setting have predictive value and may be used to assess clinical course. Scand J Infect Dis. 2008;40(2):111-20.

10. Falzon D, Schunemann HJ, Harausz E, Gonzalez-Angulo L, Lienhardt C, Jaramillo E, et al. World Health Organization treatment guidelines for drug-resistant tuberculosis, 2016 update. Eur Respir J. 2017;49(3).

11. Treatment of tuberculosis: guidelines - 4th ed. World Health Organization. 2010.

12. Gao J, Ma Y, Du J, Zhu G, Tan S, Fu Y, et al. Later emergence of acquired drug resistance and its effect on treatment outcome in patients treated with Standard Short-Course Chemotherapy for tuberculosis. BMC Pulm Med. 2016;16:26.

13. Niward K, Davies Forsman L, Bruchfeld J, Chryssanthou E, Carlström O, Alomari T, et al. Distribution of plasma concentrations of first-line anti-TB drugs and individual MICs: a prospective cohort study in a low endemic setting. J Antimicrob Chemother. 2018;73(10):2838-45.

14. Gumbo T, Chigutsa E, Pasipanodya J, Visser M, van Helden PD, Sirgel FA, et al. The pyrazinamide susceptibility breakpoint above which combination therapy fails. J Antimicrob Chemother. 2014;69(9):2420-5.

15. Kempker RR, Kipiani M, Mirtskhulava V, Tukvadze N, Magee MJ, Blumberg HM. Acquired Drug Resistance in Mycobacterium tuberculosis and Poor Outcomes among Patients with Multidrug-Resistant Tuberculosis. Emerg Infect Dis. 2015;21(6):992-1001. 
16. Forsman LD, Jonsson J, Wagrell C, Werngren J, Mansjö M, Wijkander M, et al. Minimum Inhibitory Concentrations of Fluoroquinolones and Pyrazinamide Susceptibility Correlate to Clinical Improvement in Multidrug-resistant Tuberculosis Patients: A Nationwide Swedish Cohort Study Over 2 Decades. Clinical Infectious Diseases. 2019;69(8):1394-402.

17. Horne DJ, Royce SE, Gooze L, Narita M, Hopewell PC, Nahid P, et al. Sputum monitoring during tuberculosis treatment for predicting outcome: systematic review and meta-analysis. The Lancet Infectious Diseases. 2010;10(6):387-94.

18. Schechter MC, Bizune D, Kagei M, Machaidze M, Holland DP, Oladele A, et al. Time to Sputum Culture Conversion and Treatment Outcomes Among Patients with Isoniazid-Resistant Tuberculosis in Atlanta, Georgia. Clin Infect Dis. 2017;65(11):1862-71.

19. Gegia M, Winters N, Benedetti A, van Soolingen D, Menzies D. Treatment of isoniazid-resistant tuberculosis with first-line drugs: a systematic review and meta-analysis. The Lancet Infectious Diseases. 2017;17(2):223-34.

\section{Figures}




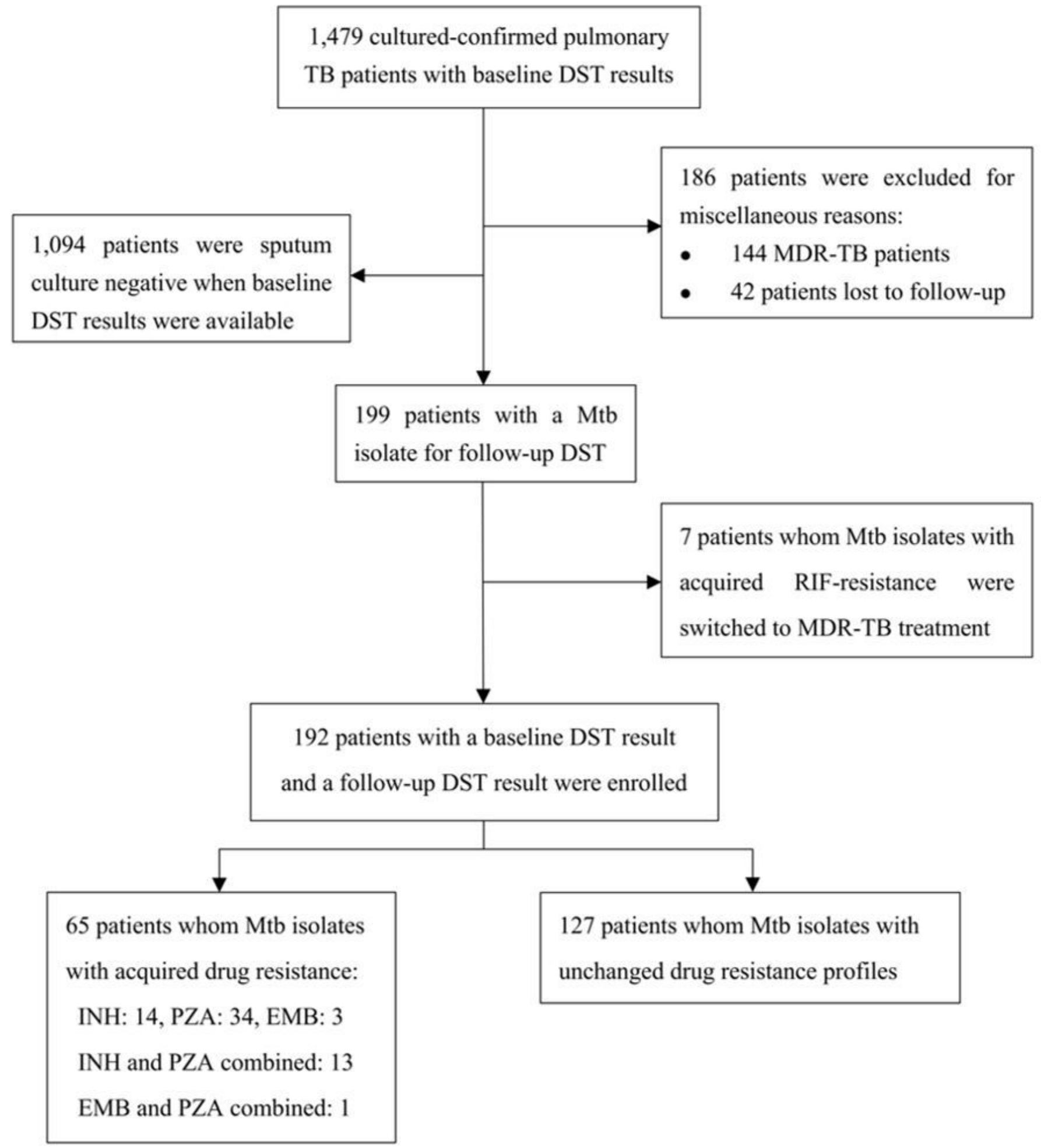

\section{Figure 1}

The enrollment of study participants. TB, tuberculosis; DST, drug susceptibility testing; TAT, turnaround time; MDR-TB, Multidrug-resistant tuberculosis; Mtb, Mycobacterium tuberculosis; RIF, rifampin; INH, isoniazid; PZA, pyrazinamide; EMB, ethambutol. 

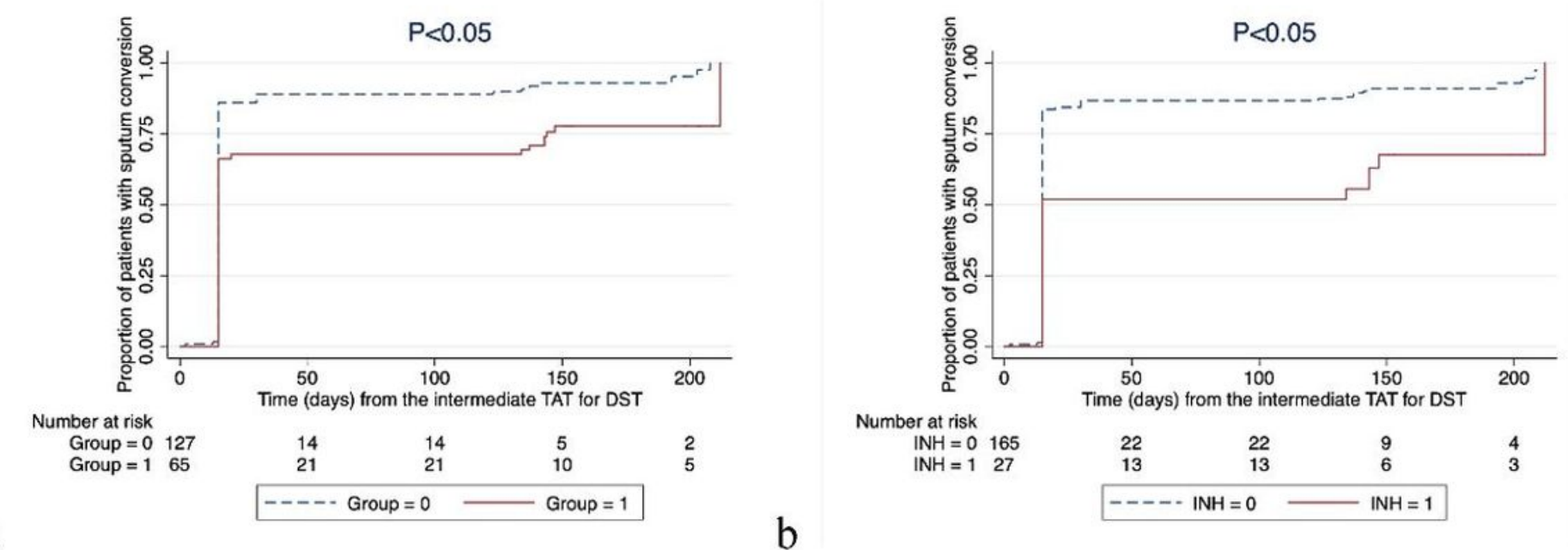

a

b
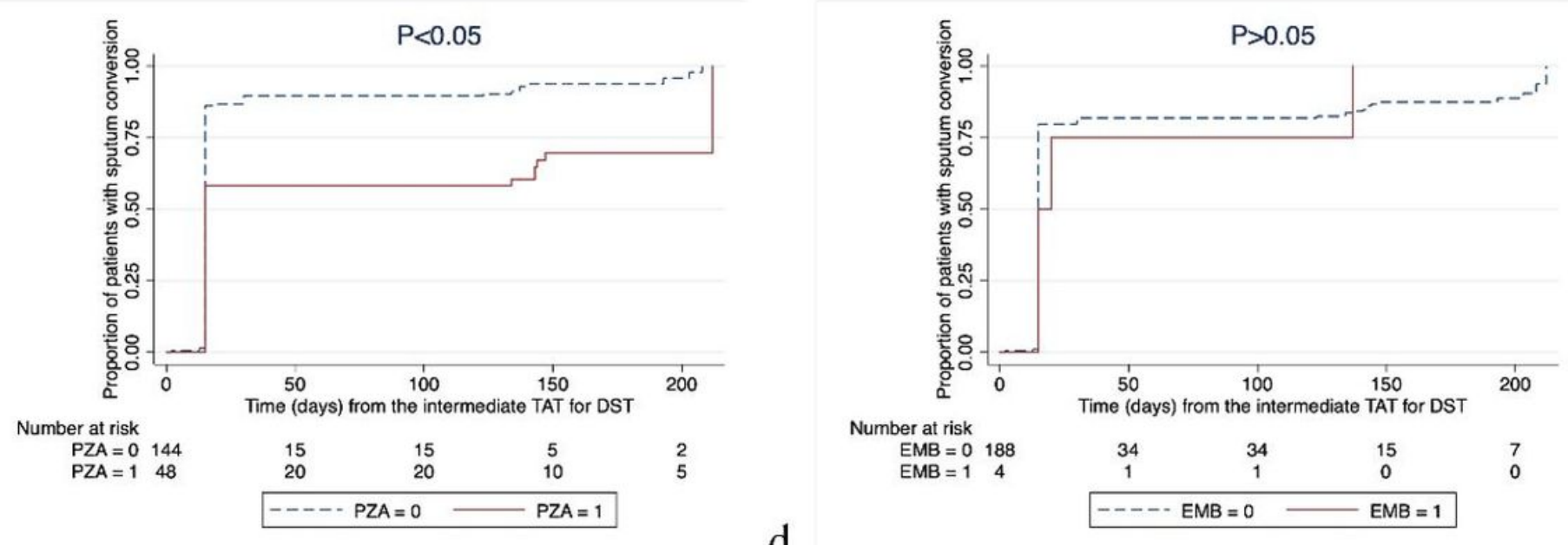

d

Figure 2

Kaplan-Meier failure curves for time from the intermediate TAT for DST to sputum culture conversion TAT for DST, turnaround time for drug-susceptibility testing; INH, isoniazid; PZA, pyrazinamide; EMB, ethambutol. Figure 2-a shows patients with acquired drug-resistant isolates (Group $=1$ ) as well as patients with unchanged drug susceptibility profile $(G r o u p=0)$. Figure 2-b shows patients with acquired INHresistant isolates $(\mathrm{INH}=1)$ as well as patients with unchanged susceptibility to $I N H(I N H=0)$. Figure 2-c shows patients with acquired PZA-resistant isolates $(P Z A=1)$ as well as patients with isolates with unchanged susceptibility to $P Z A(P Z A=0)$. Figure 2-d shows patients with acquired $E M B$-resistant isolates $(E M B=1)$ as well as patients with isolates with unchanged susceptibility to $E M B(E M B=0)$. 